\title{
Analogical Thinking Induces Eye Movements in Scene Viewing
}

\author{
Gufran Ahmad
}

\begin{abstract}
The studies of eye movements for understanding underlying mechanism of visual attention and perception have been challenging. These studies are obligatory to learn the dynamics of humans' cognition. We performed and studied eye tracking experiments to examine the hypothesis that the patterns created in scene viewing were predominantly influenced by analogical perspective of cognitive processes. Eye movement data, in terms of heat maps, were collected from participants who watched artistic portrait during active viewing. The patterns formed from eye tracking system in scene viewing mapped a series of analogical visual portions of the scene. This evidenced the hypothesis that analogical thinking induces eye movements in scene viewing.
\end{abstract}

Index Terms-Analogical thinking, eye movements, cognitive processes, scene viewing.

\section{INTRODUCTION AND BACKGROUND}

Eye tracking lies among one of the rapidly flourishing interdisciplinary research fields nowadays. People are interested in this area of study for expanding knowledge domain which is crucial to study many other areas of study. In this context, one of our previous works has been contributing to the communities of scientific, social, and commercial people as well [1].

Vision is one of the integrated activities of all human beings. Eyes are one of the senses in human body and are treated as an imperative sensor by human brain. Our eyes can move freely along all directions and by doing so, these eyes may bring human attentions, visual perceptions along with cognitive and metacognitive processes [2]-[4].

Generally, eyes engage in the verbal and nonverbal communications and convey the desirable information among the participants of communication. Further, eyes dynamically participate in the processing of data for information and the visualization of information which are common practices among professionals and individuals, including artists and scientists [2], [4]-[6].

During eye movements, eyes move to fetch a specific fraction of the visible region in viewing because of having tendency to perceive the degree of detail visible in the central direction of gaze. In the movements, they pass through two temporal phases: fixations (the stops or periods of time when

the point of gaze or significant look is relatively slow) and saccades (the hops between stopping points). Saccades are often information-seeking and directed to specific objects or

Manuscript received August 7, 2013; revised December 6, 2013. This work was supported in part by the Jazan University.

Gufran Ahmad is with the College of Computer Sciences and Information Systems, Jazan University, Jazan, Kingdom of Saudi Arabia (e-mail: gufran.researcher@gmail.com). regions by the requirements of ongoing behavior. This also concludes the existence of cognitive processes of eye movements in viewing [1], [5]-[9].

Art is characteristically human way of cognitive activity that is aimed at influencing the minds of viewers. Being constructivist in nature, it aims at the deliberate refinement and elaboration of worldviews. Most of the arts are metacognitive in its role as it engages in self-reflection, both individually and socially. The varieties and modes of art are technology-driven. Art is always aimed at realizing the cognitive consequence [10]-[12].

Analogy is basically similarity in which the same relations or likeness hold between different domains or systems. Analogy is significant in cognitive processes and is key mechanism in creativity which is also a part of the subject, like Visual Art. The main focus of analogical research is on the mapping process by which people understand one state or pattern in terms of another. Mapping is basically process of aligning the representational structures of the two cases and projecting inferences [13]-[16].

In analogical mapping, a familiar condition is used as a model for making inferences about an unfamiliar condition. The mapping process includes a structural alignment between two represented conditions and the projection of inferences from one to the other. The alignment must be structurally consistent, i.e. there must be a one-to-one correspondence between the mapped elements in the base and target, and the arguments of corresponding predicates must also correspond [13]-[16].

This also lays the foundation of analogical thinking that gets evolved during the phenomena of analogy. If analogy is thought, then analogical thinking may be considered as flow of analogical thought. Principally, analogical thinking is a process that takes analogy as an idea into consideration [13]-[16].

During artistic portrait observation, we move our eyes rapidly in irregular manner to change focus from one fixation to another fixation. This process, saccade, is one of the most common behavior of eyes. Pattern is obtained only during the periods of relative gaze constancy, known as fixations. The process of directing the eyes to view picture in real time is known as gazing of eyes [6]-[9], [17]-[19].

The main motive of this study in artistic scene viewing is to identify and illustrate the idea of analogy and the process of analogical thinking which is stimulated by the cognitive and metacognitive processes that are observed by the shifts of gaze during the eye movements.

\section{EYE TRACKING SYSTEM}

In eye tracking system, the system illuminates infrared light 
for tracking the eye movements. The camera, connected to the system, captures the location of viewer's eyes in terms of fixation during experimentation time. As the viewer moves his/her eyes to look a new location of the scene, the camera records new fixation also. This process of recording continues subsequently. The system generates eye tracks and heat maps using the captured data which is utilized for further analysis.

The schematic diagram of eye tracking system and basic processes involved during eye tracking experimentation is represented in Fig. 1.

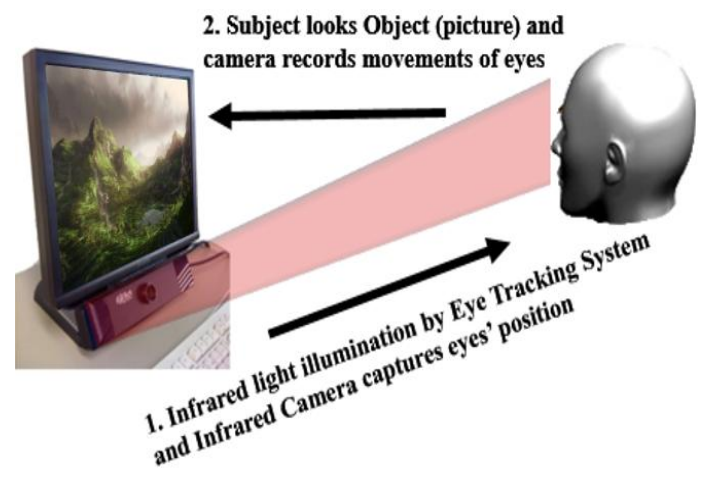

Fig. 1. Eye tracking system with operational processes.

The traces of eye movements are taken in diverse layouts as per analyst's suitability. Among them, there are two most common formats are Heat Map and Sequenced Gazing with circle of concentration. In Heat Map, the track of eye is recorded as illumination and intensity of infrared light rays. This is based on Energy Therapy Technique (ETT). In Sequenced Gazing, the eye tracks are entered as numbered circles with their areas indicating the time duration of eye's gazing in those areas respectively [3], [5].

In our experiments, we study the heat maps of viewer's eye movements, which is generated by the system, during scene viewing. These are assured evidences of eye movements.

\section{PRESENT STUDY}

We investigate the eye movements from cognitive perspective during scene viewing and analyze the patterns of heat map to visualize the information. Here, visualization of heat map patterns to extract information for interpretation are essential involvements of this study.

Initially, eye movement heat map was collected from participants who viewed full-color scenes while engaged in a visual search task in which they are freely viewing different regions appeared in each picture. Finally, we compared and analyzed the heat map against the artistic portrait. The interpretation is carried out with the help of cognitive and metacognitive processes in current research.

\section{FLOW CHART OF STUDY}

The study on eye movements during scene viewing consists of a number of steps to be performed. These steps are represented as shown in the adjacent flow diagram (Fig. 2). This is a comparative study of two items; one item is artistic portrait and other item is the heat map of the same portrait, which generated from eye tracking system.

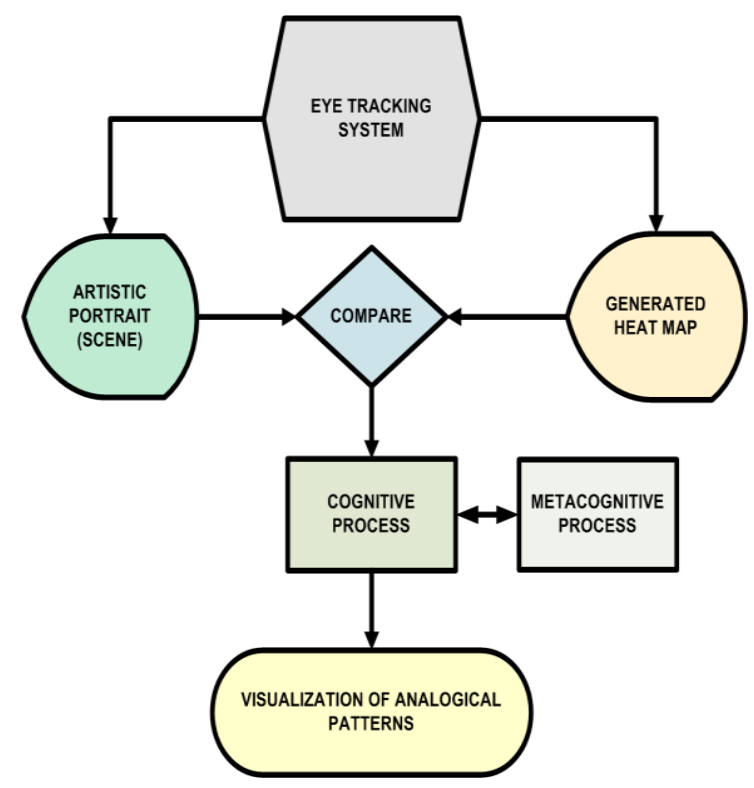

Fig. 2. Flow chart of research study.

It begins with recording of heat map of eye movements for a Subject; a viewer on eye tracking system for an Object; artistic portrait. The generated heat map of the same artistic portrait is comparable to the original artistic portrait. This comparative analysis brings visualization and interpretation of the outcome. So, these two items gets compared side by side.

During comparison stage, we utilize cognitive process, and metacognitive process, in addition to analogical perspective, to understand the hidden mechanism that creates resultant maps. By analyzing, we come up with concluding remarks on evolving phenomena.

\section{METHOD}

We selected 38 participants from a number of fields within university, aging from 22 years to 32 years. These Subjects, the participants were assigned to view 3 randomly selected famous artistic portraits as shown below in Fig. 3.

The artistic portraits are "Green Hills", "Autumnal scenery in the evening", "Artistic expressions".
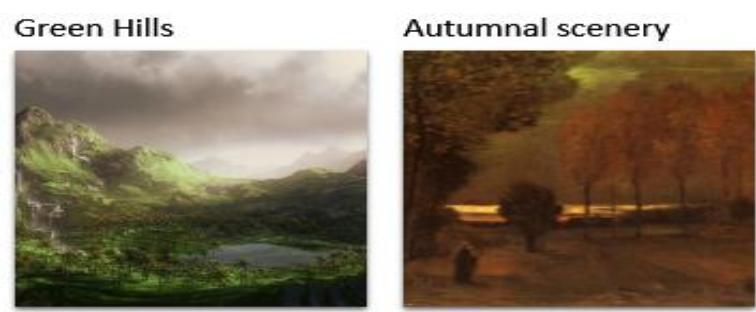

Artistic Expressions

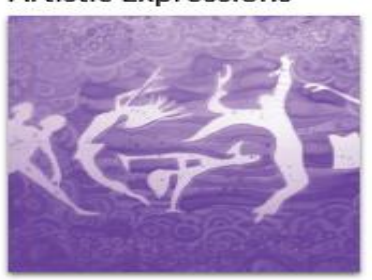

Fig. 3. Selected artistic portraits for research study. 
Their eye movements were closely monitored as they viewed 32 bits full-color artistic pictures. The Objects, the pictures were displayed on a computer monitor. The pictures were shown at a resolution of $1280 \times 1024$ pixels and subtended $15 \mathrm{deg}$. horizontally by $10 \mathrm{deg}$. vertically at a viewing distance of $75 \mathrm{~cm}$. Eye position was sampled from an Eye Tech Digital Systems TM3 16 mm Eye Tracker, and eye tracking data was parsed into fixations within heat maps.

The Subject's head was held steady in advance prior to experimentation. Prior to the first trial, Subjects completed a procedure to calibrate the output of the eye tracker against spatial positions on the display screen. This procedure was repeated regularly throughout the experiment to maintain high level of accuracy. Subjects were initiated to view the pictures freely.

The pictures were presented to the Subjects for maximum duration of $100 \mathrm{sec}$. During this time span, the Subjects viewed the pictures with their normal eyes and focused attention on the Object, the portrait.

Here, we analyzed all 3 portraits respectively with the intention to elaborate our findings in the most common and generalized perspective.

\section{Analysis 1: Study of Green Hills Artistic Portrait}
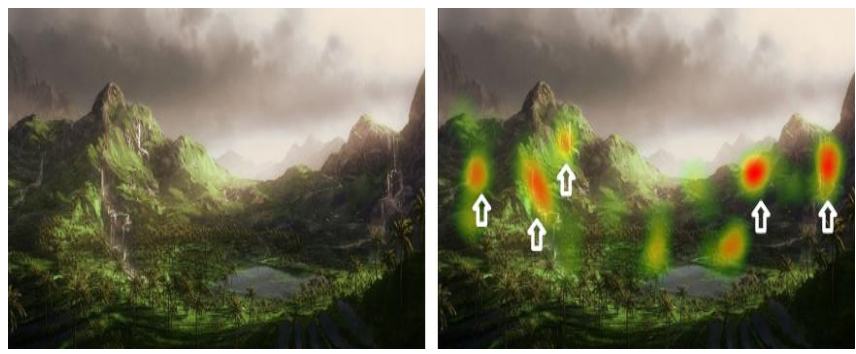

Fig. 4. Portrait and generated heat map of the same portrait.

In the above artistic portrait of Fig. 4, the left side portrait is the original portrait consisting of Hills, central Lake, dark Clouds, Palm trees, and Waterfalls. The right side portrait is the heat map generated by eye tracking system during the scene viewing by the Subject.

Subjects viewed at different regions of the portrait like central Lake, Hills, and finally, Waterfalls. As soon as they came across the region of Waterfall, their visual attention cognitively fell into the process of perception. Gradually, they moved to adjacent region of the Waterfall because of the ongoing cognitive processes in scene viewing [1], [6], [7], [9]-[12], [17]-[19]. So, the attention of the Subjects shifted to another similar region of the portrait, i.e. another Waterfall, because of the associated analogical thinking that came into play in the middle of the initiated cognitive process [13]-[16].

With this process of analogical thinking, the Subjects got motivated to move subsequently to other similar regions of the portrait, which is obvious from the heat map of the portrait Thus, they viewed a number of Waterfalls subsequently.

The idea of analogy and the process of analogical thinking are the core features in this phenomenon of scene viewing experimentation. These led to the visualization of analogical patterns which were generated by the eye movements of the Subject. These processes of analogical thinking continued repeatedly during the entire span of viewing time.
Further, later interview of Subjects confirmed that they got interest in central Lake, then Hills, and finally, were very excited to see a number of Waterfalls which were at the left side as well as right side of the artistic portrait.

\section{Analysis 2: Study of Autumnal Scenery Artistic Portrait}
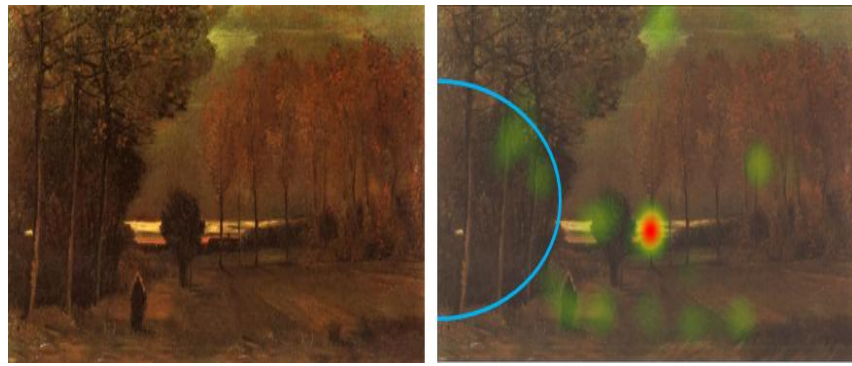

Fig. 5. Portrait and generated heat map of the same portrait.

In this artistic portrait of Fig. 5, the Subjects gazed at the artistic portrait "Autumnal scenery in the evening" by Van Gogh. The portrait was filled with shiny background with riverbank, glossy trees, sunlit field, and gleaming cloud, etc.

In this experimentation, the Subjects viewed mostly central, lower, and upper regions of the portrait. Here, the Subjects' visual attentions and consciousness caused Subjects' mind towards initiation of cognitive processes [1], [6], [8]-[12], [18], [19]. These cognitive processes perceived knowledge of different regions of the portrait. This ultimately, originated the awareness of analogy and in turn, the process of analogical thinking based on brightness contrast of the regions. The left semi-circled region, the central tree, and the human are a few elements which were relatively darkened in the portrait.

The illuminating sunshine caused most of portrait's regions filled with high intensity of light. Thus, the Subjects got influenced by this heavily shining regions of the portrait and viewed in these regions analogically.

In successive interview, the Subjects stated that they were strongly induced by the brightness contrast in scene viewing. Mostly, they was interested in viewing all brightly visible portions of portrait.

\section{Analysis 3: Study of Artistic Expressions Portrait}
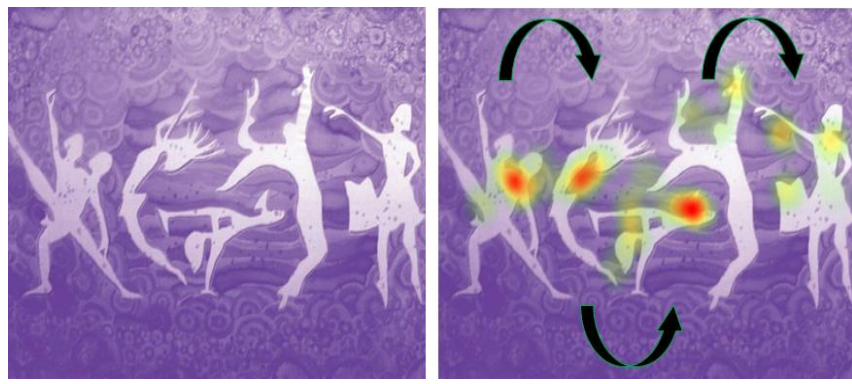

Fig. 6. Portrait and generated heat map of the same portrait.

In the Artist's Expression portrait of Fig. 6, the portrait was filled with a number of Artist's Expressions for individuals. The Artist communicated his Expressions in terms of gestures and acts. This also involved animals in addition to, humans.

During experimentation of eye movements, Subjects were mostly gazing at different regions of the portrait. Visual attention at these different elements of the portrait were focused for underlying perceptual theme. The acts and 
gestures of these living creatures communicated the causal context by visual perception. All these actions of joy and happiness that were performed by everyone in the portrait got Subjects' consciousness finally [1], [4], [6]-[12], [17], [18].

Thus, the initiated cognitive processes of the Subjects' minds stimulated emotional feelings and sentiments related with these living creatures. Further, these attributes turned into a logical basis of analogy and the process of analogical thinking mapped these living creatures together to relate with one another.

Later interview of the Subjects concluded that they were interested in viewing living creatures and their emotional feelings and concerned activities of these living creatures. They felt comparative similarity for these behaviors among these living creatures.

\section{DISCUSSION}

In this study, the artistic portraits broaden their impact from the fact that the artists are visualizing this pragmatic world in different standpoint, i.e. humanitarian standpoint. These emotional viewpoints are rather too complicated to comprehend from visual analytics and analytical reasoning. These cognitive perspectives and intrinsic analogical thinking are revealed by eye movements during scene viewing.

During the last stage of experiments, the visualization of analogical patterns is a crucial and central part of whole activity. The visualization of analogical patterns are undeniably astonished perspectives of each and every analyst who inspect them for the definite intents. This, in turn, brings a number of analogies in scene viewing by various perspectives of analysts. Though the existence of analogical thinking is available in the literature [11]-[16], yet its presence and explanation varies drastically. In this regard, the analogical patterns reinforce again the existence of analogical thinking in between the inherent cognitive and metacognitive processes.

Shifting (saccades) and gazing during scene viewing are decisive factor for suitable achievement of task-relevant visual information [6], [9], [17]-[19]. In this study, we observed that the generated heat maps by eye movements are significant and are primary evidences to prove the analogical thinking and associated cognitive processes in eye movements. These analogical thinking are able to express human's cognitive aspects in terms of illumination and intensity observed in the heat maps. Without taking the account of analogical thinking into consideration, it is impossible to understand the underlying sequential cognitive and metacognitive aspects as well.

Although there are a few biased shifts in eye movements during experimentations because of highly influential factors like emotions, unintentional shifts just before gaining visual attentions, or even flow of another thought in between, yet these cannot be taken into consideration as being negligible amount. On the contrary, this is also a persuasive evidence for understanding about analogical thinking as how dominant these are. Despite getting biased by other factors and processes, these analogical thinking processes never get any halt. Active scene viewing gets stimulated by these mighty flow of thoughts which is based on concept of analogy.

Our final remark summarizes that the experimental evidence of analogical thinking and generated analogical patterns during active scene viewing supports our hypothesis for which we conducted a series of experimentations. The hypothesis that analogical thinking induces eye movements in scene viewing, is the only and obvious demonstration related to eye movements research study.

\section{CONCLUSION}

Our findings suggested the existence of analogical thinking of the human mind in the middle of cognitive and metacognitive processes which were continuously flowing during active scene viewing. These analogical thinking led to the creation of analogical patterns of scene's elements.

Moreover, these existed analogical thinking were found to be based on contexts of the Object and might vary depending on the Subjects' visual attention and analogical perception. Nonetheless, analogical thinking mostly, were integral part of humans' cognitive processes.

\section{FUTURE PLAN}

Two major future works are needed to be carried out related to the current work.

There are a number of models related to human mind that have been proposed so far. However, none of them could satisfactorily answers issues related to humans' cognitive processes in relation to analogical thinking during visual perception. So, the creation of such model is one of the directions of future study.

Generalization of humans' analogical perception has been one of the foremost challenges in the area of cognitive science. This task of generalization is to be extended in future eye movement studies.

\section{ACKNOWLEDGMENT}

We sincerely acknowledge all the participants who gave their valuable time for experimentations. Also, the university and financial supporters are highly appreciated for this creative piece of work.

\section{REFERENCES}

[1] G. Ahmad, Y. Ohsawa, and Y. Nishihara, "Cognitive impact of eye movements in picture viewing," International Journal of Intelligent Information Processing, vol. 2, no. 1, 1-8. 2011.

[2] K. Rogers, The Eye: The Physiology of Human Perception. NY: Britannica Educational Publishing, 2010.

[3] T. Brown, The Science and Art of Tracking, Y: Berkley Books, 1999.

[4] A. Ells. Emotional Disorders and Metacognition: Innovative Cognitive Therapy, West Sussex: John Wiley \& Sons, 2000.

[5] A. T. Duchowski, Eye Tracking Methodology: Theory and Practice. London: Springer Verlag, 2003.

[6] J. Holsanova, Discourse, Vision, and Cognition, John Benjamins Publishing Company, 2008.

[7] Z. M. Griffin, "Why look? reasons for eye movements related to language production," in The Integration of Language, Vision, and Action: Eye Movements and the Visual World, Henderson and Ferreira, New York: Psychology Press, 2004, pp. 213-247.

[8] J. M. Henderson and A. Hollingworth, "High level scene perception," Annual Review of Psychology, vol. 50, pp. 243-271, 1999. 
[9] K. Rayner, Eye movements and Visual Cognition: Scene Perception and Reading, New York: Springer Verlag, 1992.

[10] F. B. Viegas and M. Wattenberg, "Artistic data virtualization: beyond visual analytics," in Proc. Online Communities and Social Computing, Second International Conference, Beijing: Springer, 2007, pp. 182-191.

[11] M. Turner, The Artful Mind - Cognitive Science and the Riddle of Human Creativity, Oxford University Press, 2006.

[12] R. L. Solso, Cognition and Visual Arts, Massachusetts London: MIT Press, 1994.

[13] D. Gentner, "Structure-mapping: A theoretical framework for analogy," Cognitive Science, vol. 7, pp. 155-170, 1983.

[14] D. Gentner and A. B. Markman, "Structure-Mapping in Analogy and Similarity," American Psychologist, vol. 52, pp. 45-56, 1997.

[15] D. Gentner, M. J. Rattermann, and K. D. Forbus. "The roles of similarity in transfer: separating retrievability from inferential soundness." Cognitive Psychology, vol. 25, pp. 524-575, 1993.

[16] D. Gentner and J. Medina. "Similarity and the development of rules," Cognition, vol. 65, pp. 263-297, 1998

[17] J. M. Henderson and A. Hollingworth. "Eye movements during Scene viewing: an overview," in Eye Guidance in Reading and Scene Perception, G. W. Underwood, Ed. Oxford: Elsevier, 1998, pp. 269-293.

[18] J. E. Hoffman, "Visual Attention and Eye Movements," in Attention, H Pashler, Ed. London: Psychology Press, 1998, 119-153.

[19] L. Stark and S. R. Ellis, "Scanpaths revisited: cognitive models direct active looking," in Eye Movements: Cognition and Visual Perception, D. F. Fisher, R. A. Monty and, J. W. Senders, Eds. Hillsdale: Lawrence Erlbaum Associates, 1981, pp. 193-226.

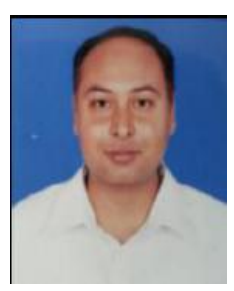

Gufran Ahmad has experience in teaching, research and industrial domains for national and international communities. He is an ardent thinker and passionate knowledge sharer for cultivating intelligence among Scholars and Learners.

$\mathrm{He}$ is dedicating his exertion on visualization, intelligent information processing, predictive modeling, and decision making which are hot talking points of KDD, AI, Cognitive Science, and HCI, not only from research perspective but from industrial perspective also.

He holds master of engineering in computer software and theory from Harbin Engineering University, China, Postgraduate Diploma in computer applications from Jamia Millia Islamia, India, master of science in theoretical physics from Jamia Millia Islamia, India, bachelor of science in physics from Jamia Millia Islamia, India.

$\mathrm{He}$ is currently a faculty and researcher for College of Computer Sciences and Information Systems, Jazan University. Earlier, he devoted his endeavor as Researcher and TF in The University of Tokyo, Faculty in Al-Falah Engineering College, Lecturer in Institute of Technology, Researcher and Teaching Assistant in Mie University, Japan, and IT Professional in Fintech Compu Systems Limited \& Micro Computer Services \& Solutions companies.

$\mathrm{He}$ collaborated and wrote national and international articles, publications, teaching and technical notes that can demonstrate his writing intents. These creativities were appended as valued assets by organizations and corporates.

Mr. Ahmad has been a committed member of Physics Association, National Computer Software Society, AICIT, and National Literacy Mission. He received numerous awards, including Best Employee of Corporate, Certificate of Merit, Best Author, and Young Researcher. 